PROCEEDINGS OF THE

AMERICAN MATHEMATICAL SOCIETY

Volume 133, Number 7 , Pages 1897-1905

S 0002-9939(05)07739-7

Article electronically published on February 15, 2005

\title{
THE HOMOLOGICAL DETERMINANT OF QUANTUM GROUPS OF TYPE $A$
}

\author{
PHÙNG HỒ HAI \\ (Communicated by Martin Lorenz)
}

\begin{abstract}
Let $R$ be a Hecke symmetry depending algebraically on a parameter $q \in \mathbb{C}$. We show that the homology of the Koszul complex associated with $R$ is one-dimensional when $q$ is not a root of unity. A generator of this homology group then induces the homological determinant of the quantum group associated with $R$.
\end{abstract}

\section{INTRODUCTION}

Let $V$ be a vector space over a field $k$ and $G L(V)$ the general linear group. It is well known that elements of $G L(V)$ act on the $n$-th homogeneous component of the exterior algebra over $V$ by means of the determinant. More precisely, let $x_{1}, x_{2}, \ldots, x_{d}$ be a basis of $V$. Then $\wedge_{d}(V)$ is one-dimensional and a non-zero vector is $x_{1} \wedge x_{2} \wedge \ldots \wedge x_{d}$. If $g \in G L(V)$ has the matrix $A$ with respect to this basis, then

$$
g \cdot\left(x_{1} \wedge x_{2} \wedge \ldots \wedge x_{d}\right)=\operatorname{det} A \cdot x_{1} \wedge x_{2} \wedge \ldots \wedge x_{d} .
$$

Now let $V$ be a vector superspace of dimension $(r \mid s), r+s=d$. The super group $G L(V)$ is defined as follows. Let $x_{1}, x_{2}, \ldots, x_{d}$ be a homogeneous basis of $V$, where the parity of the first $r$ elements is even and the parity of the rest is odd. Let $z_{j}^{i}$ be the endomorphism that maps $x_{i}$ to $x_{j}$ and other basis elements to zero. We consider $z_{j}^{i}$ as a generator with parity being the sum of those of $x_{i}$ and $x_{j}$. The super semi-group $\operatorname{End}(V)$ is the spectrum of the super commutative algebra

$$
M:=k\left\langle\left\{z_{j}^{i}\right\}_{1 \leq i, j \leq d}\right\rangle /\left(z_{j}^{i} z_{l}^{k}=(-1)^{(\hat{i}+\hat{j})(\hat{k}+\hat{l})} z_{l}^{k} z_{j}^{i}\right)
$$

(where $k\left\langle\left\{z_{j}^{i}\right\}_{1 \leq i, j \leq d}\right\rangle$ denotes the free non-commutative algebra and $\hat{i}$ denotes the parity of $x_{i}$ ). Thus, for a super commutative algebra $K$, an endomorphism of $V_{K}:=V \otimes K$ is a $K$-point of $M$, i.e. an algebra homomorphism $M \longrightarrow K$.

The invertibility of a super matrix can be given in terms of the super determinant or Berezinian, which was introduced by Berezin. Let $K$ be a super commutative algebra and $Z$ be a $K$-point of $\operatorname{End}(V)$. The matrix $Z=\left(z_{j}^{i}\right)$ has the following form: $Z=\left(\begin{array}{ll}A & B \\ C & D\end{array}\right)$ where $A, D$ are square matrices of dimension $m \times m$ and $n \times n$, respectively, whose entries' parities are even, and $B, D$ are matrices of types $m \times n$

Received by the editors September 19, 2002 and, in revised form, February 22, 2004.

2000 Mathematics Subject Classification. Primary 16W30, 17B37; Secondary 17A45, 17A70.

This work was supported by the National Program of Basic Sciences Research of Vietnam. 
and $n \times m$, whose entries' parities are odd. The super determinant of $Z$ is defined to be

$$
\operatorname{Ber} Z=\operatorname{det} T^{-1} \operatorname{det}\left(A-C D^{-1} B\right) .
$$

It is shown that the matrix $Z$ is invertible iff its super determinant is and that the super determinant is multiplicative. Thus, the invertible super matrix forms a group $G L(V)$, which is an algebraic super-subgroup of $\operatorname{End}(V)$. It is however not clear why the definition of Ber is independent of the choice of bases (our basis is a distinguished basis).

In [17] Manin suggested the following construction to define the super determinant. Let $V^{*}$ denote the vector space dual to $V$ with the dual basis $\xi^{1}, \xi^{2}, \ldots, \xi^{n}$, $\xi^{i}\left(x_{j}\right)=\delta_{j}^{i}$. Manin introduced the following Koszul complex: its $(k, l)$-term is given by $K^{k, l}:=\wedge_{k} \otimes \mathrm{S}_{l}{ }^{*}$, where $\wedge_{n}$ and $\mathrm{S}_{n}$ are the $n$-th homogeneous components of the exterior and the symmetric tensor algebra over $V$. The differential $d^{k, l}: K^{k, l} \longrightarrow K^{k+1, l+1}$ is given by

$$
d^{k, l}(h \otimes \phi)=\sum_{i} h x_{i} \otimes \xi^{i} \wedge \phi
$$

It is easy to check that $d_{k, l}$ is $G L(V)$-equivariant; hence the homology groups of this complex are representations of $G L(V)$. On the other hand, one can show that this complex is exact everywhere except at the term $(m, n)$, where the homology group is one-dimensional; thus, it defines a one-dimensional representation of $G L(V)$. It turns out that elements of $G L(V)$ act on this representation by means of its super determinant; in other words, the definition of the super determinant is basis free.

The quantum semigroup of type $A$ is the "spectrum" of the bialgebra

$$
E:=k\left\langle\left\{z_{j}^{i}\right\}_{1 \leq i, j \leq d}\right\rangle /\left(R_{u v}^{i j} z_{k}^{u} z_{l}^{v}=z_{t}^{i} z_{s}^{j} R_{k l}^{t s}\right)
$$

where $R$ is a Hecke symmetry (see $₫ 1$. The Hecke symmetry resembles the usual flipping operator $a \otimes b \longmapsto b \otimes a$ or $a \otimes b \longmapsto(-1)^{\hat{a} b} b \otimes a$ ( $a, b$ are homogeneous) in super symmetry.

In [5, 14, a Koszul complex is defined for $R$. For that, one first has to define the quantum exterior and quantum symmetric tensors by means of certain projectors on $V^{\otimes n}$. It is still an open question whether this complex has the homology group concentrated at a certain term and its dimension is one. Some efforts have been made. Gurevich [5] showed this for even Hecke symmetries (i.e., those that induce a finite-dimensional exterior algebra); Lyubashenko and Sudbery [14] showed this for Hecke sums of an odd and an even Hecke symmetry.

In this paper, assuming that $R$ depends algebraically on $q$, where $q$ runs in $\mathbb{C}$, we give the affirmative answer to this question for an algebraically dense set of values of $q$. Our tactic is first to use a new result of Deligne [1] to check the case $q=1$. Then using a standard argument we show that for a dense set of values of $q$, the homology group of $K$ has dimension less than that of the corresponding homology groups when $q=1$. In other words, for an algebraically dense subset of $\mathbb{C}$, the homology group has dimension at most 1 . It remains to show the non-vanishing of the homology. 


\section{HeCKe Symmetries AND THE ASSOCIATED QUANTUM GROUPS}

We work over an algebraically closed field $k$ of characteristic zero. Let $V$ be a vector space over $k$ of dimension $d$. Let $R: V \otimes V \longrightarrow V \otimes V$ be an invertible operator. $R$ is called a Hecke symmetry if the following conditions are fulfilled:

- $R_{1} R_{2} R_{1}=R_{2} R_{1} R_{2}$, where $R_{1}:=R \otimes \mathrm{id}_{V}, R_{2}:=\mathrm{id}_{V} \otimes R$,

- $(R+1)(R-q)=0$ for some $q \in k$.

- The half adjoint to $R, R^{\sharp}: V^{*} \otimes V \longrightarrow V \otimes V^{*},\left\langle R^{\sharp}(\xi \otimes v), w\right\rangle=\langle\xi, R(v \otimes w)\rangle$, is invertible.

Throughout this work we will assume that $q$ is not a root of unity other than the unity itself. If $q=1, R$ is called vector symmetry. Vector symmetries were introduced by Lyubashenko [13] and generalized to Hecke symmetries by Gurevich [5].

Let us fix a basis $x_{1}, x_{2}, \ldots, x_{d}$ of $V$. Then $R$ can be given in terms of a matrix, also denoted by $R, R\left(x_{i} \otimes x_{j}\right)=x_{k} \otimes x_{l} R_{i j}^{k l}$, where we adopt the convention of summing over the indices that appear in both the lower and upper places. The matrix $R^{\sharp}{ }_{i j}^{k l}$ is given by $R_{i j}^{\sharp k l}=R_{j l}^{i k}$. Therefore, the invertibility of $R^{\sharp}$ can be expressed as follows: there exists a matrix $P$ such that

$$
P_{j n}^{i m} R_{m l}^{n k}=\delta_{l}^{i} \delta_{j}^{k}, \quad R_{j n}^{i m} P_{m l}^{n k}=\delta_{l}^{i} \delta_{j}^{k} .
$$

Consider the following algebra:

$$
E_{R}:=k\left\langle\left\{z_{j}^{i}\right\}_{1 \leq i, j \leq d}\right\rangle /\left(z_{m}^{i} z_{n}^{j} R_{k l}^{m n}=R_{p q}^{i j} z_{k}^{p} z_{l}^{q}\right),
$$

which is in fact a coquasitriangular bialgebra [12, 13. with the coproduct given by $\Delta\left(z_{j}^{i}\right)=z_{k}^{i} \otimes z_{j}^{k}$ and the counit given by $\varepsilon\left(z_{j}^{i}\right)=\delta_{j}^{i}$. The coquasitriangular structure is given by $r\left(z_{j}^{i}, z_{l}^{k}\right)=R_{j l}^{k i}$. The bialgebra $E$ is called the "function algebra" on the corresponding quantum endomorphism space or the matrix quantum semigroup.

There is a right coaction of $E_{R}$ on $V$, given by $\delta\left(x_{i}\right)=x_{j} \otimes z_{i}^{j}$. This coaction induces actions of $E_{R}$ on $V^{\otimes n}$ for $n \geq 1$. The braiding on $V \otimes V$ induced from the coquasitriangular structure $r$ is precisely the operator $R$. There is a natural $\mathbb{N}$-grading on $E_{R}$, where the $n$-th homogeneous component consists of homogeneous polynomials of total degree $n$ and is denoted by $E_{n}$. Then $E_{n}$ is a coalgebra and coacts on $V^{\otimes n}$ from the right; hence its dual $E_{n}^{*}$ acts on $V^{\otimes n}$ from the left.

The Hecke algebra $\mathcal{H}_{n}=\mathcal{H}_{q, n}$ has generators $T_{i}, 1 \leq i \leq n-1$, subject to the relations: $T_{i} T_{j}=T_{j} T_{i},|i-j| \geq 2 ; T_{i} T_{i+1} T_{i}=T_{i+1} T_{i} T_{i+1}, 1 \leq i \leq n-2 ; T_{i}^{2}=$ $(q-1) T_{i}+q$. There is a $k$-basis in $\mathcal{H}_{n}$ indexed by permutations of $n$ elements: $T_{w}, w \in \mathfrak{S}_{n}\left(\mathfrak{S}_{n}\right.$ is the permutation group), in such a way that $T_{(i, i+1)}=T_{i}$ and $T_{w} T_{v}=T_{w v}$ if the length of $w v$ is equal to the sum of the length of $w$ and the length of $v$. If $q$ is not a root of unity of degree greater than $1, \mathcal{H}_{n}$ is a semisimple algebra. For more details, the reader is referred to [2, 3].

The Hecke symmetry $R$ induces an action of the Hecke algebra $\mathcal{H}_{n}=\mathcal{H}_{q, n}$ on $V^{\otimes n}, T_{i} \longmapsto R_{i}=\mathrm{id}^{i-1} \otimes R \otimes \mathrm{id}^{n-i-1}$ that commutes with the coaction of $E_{R}$. The action of $T_{w}$ will be denoted by $R_{w}$. We have the following "Double centralizer theorem" [6, Thm. 2.1].

1.1. The algebras $\rho_{n}\left(\mathcal{H}_{n}\right)$ and $E_{n}^{*}$ are centralizers of each other in $\operatorname{End}_{k}\left(V^{\otimes n}\right)$.

Consequently, simple $E_{n}^{*}$-modules (and hence simple $E_{n}$-comodules) can be given as the image of primitive idempotents of $\mathcal{H}_{n}$, and conjugate idempotents determine isomorphic (co)modules. Since conjugate classes of primitive idempotents of $\mathcal{H}_{n}$ are 
indexed by partitions of $n$, simple subcomodules of $V^{\otimes n}$ are indexed by a subset of partitions of $n$. Thus $E$ is cosemisimple, and its simple comodules are indexed by a subset of partitions.

Let $I_{\lambda}$ denote the simple comodule corresponding to the partition $\lambda$. Then $I_{\lambda}$ and $I_{\mu}$ can be realized as the images of two primitive idempotents $e_{\lambda} \in \mathcal{H}_{r}$ and $e_{\mu} \in \mathcal{H}_{s}$. Thus $I_{\lambda} \otimes I_{\mu}$ is the image of a (not necessarily primitive) idempotent in $\mathcal{H}_{r+s}$. This idempotent decomposes into an orthogonal sum of primitive idempotents, which yields a decomposition of $I_{\lambda}$ and $I_{\mu}$ into a direct sum of simple subcomodules. Taking into account that conjugate idempotents define isomorphic comodules, we have 7

$$
I_{\lambda} \otimes I_{\mu} \cong \bigoplus_{\gamma} I_{\gamma}^{\oplus c_{\lambda \mu}^{\gamma}}
$$

where the $c_{\lambda \mu}^{\gamma}$ are the Littlewood-Richardson coefficients describing the multiplicity of the Schur function $s_{\gamma}$ in the product of two other Schur functions $s_{\lambda}$ and $s_{\mu}$ (cf. [15]).

Example (Quantum symmetrizers). The primitive idempotent

$$
X_{n}:=\frac{1}{[n]_{q}} \sum_{w \in \mathfrak{S}_{n}} R_{w}
$$

determines a simple comodule $\mathrm{S}_{n}$ called the $n$-th quantum symmetric tensor power, and the primitive idempotent

$$
Y_{n}:=\frac{1}{[n]_{1 / q}} \sum_{w \in \mathfrak{S}_{n}}(-q)^{-l(w)} R_{w}
$$

determines a simple comodule $\wedge_{n}$ called the $n$-th quantum anti-symmetric tensor power. Notice that $\mathrm{S}_{n}=I_{(n)}$ and $\wedge_{n}=I_{\left(1^{n}\right)}$.

Let us briefly recall here the Littlewood-Richardson algorithm for computing the coefficients $c_{\lambda \mu}^{\gamma}$ [15]. Let $\gamma$ and $\lambda$ be partitions with $\gamma_{i} \geq \lambda_{i}$ for all $i$. We define the skew diagram $[\gamma \backslash \lambda]:=\left\{(i, j):(i, j) \in[\gamma], \lambda_{i}<j \leq \gamma_{i}\right\}$. The $i$-th row of the diagram consists of nodes $(i, j)$ with fixed $i$, and the $j$-th column consists of nodes $(i, j)$ with fixed $j$.

Let $\mu$ be a partition. A sequence of positive integers is said to have type $\mu$ if each $i$ occurs $\mu_{i}$ times. Such a sequence is said to be "good" if for any term $i>1$, the number of previous $i-1$ in the sequence is strictly greater than the number of previous $i$. For example, the good sequences of type $\mu=(2,1)$ are 112,121.

The coefficient $c_{\lambda \mu}^{\gamma}$, where $\lambda$ is a partition of $r, \mu$ is a partition of $s$ and $\gamma$ is a partition of $r+s$, can be computed as follows:

(i) if $\lambda_{i}>\gamma_{i}$ for some $i$, then $c_{\lambda \mu}^{\gamma}=0$;

(ii) if $\lambda_{i} \leq \gamma_{i}$ for every $i$, then $c_{\lambda \mu}^{\gamma}$ is the number of ways of replacing the nodes $(i, j)$ of $[\gamma \backslash \lambda]$ by integers, such that

- each $k$ occurs $\mu_{k}$ times;

- the numbers are non-decreasing along rows and strictly increasing down columns;

- when reading from right to left in successive rows, we have a good sequence of type $\mu$. 
Example. Let $[\lambda]=\bigoplus$ and $[\mu]=\bigoplus$. There are two good sequences of type $\mu=(2,1)$ : (112), (121). We have the following possibilities for $\gamma$ for which $c_{\lambda \mu}^{\gamma} \neq 0$ :

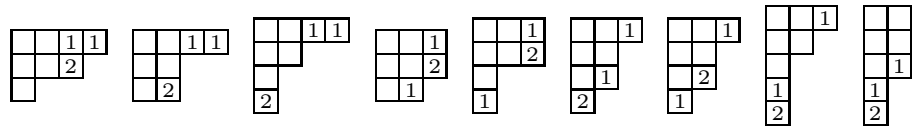

which means

$$
\begin{aligned}
I_{(2,2,1)} \otimes I_{(2,1)}=I_{(4,3,1)} & \oplus I_{\left(4,2^{2}\right)} \oplus I_{\left(4,2,1^{2}\right)} \oplus I_{\left(3^{2}, 2\right)} \oplus I_{\left(3^{2}, 1^{2}\right)} \oplus I_{\left(3,2^{2}, 1\right)} \oplus 2 \\
& \oplus I_{\left(3,2,1^{3}\right)} \oplus I_{\left(2^{3}, 1^{2}\right)} .
\end{aligned}
$$

Note however that not every partition defines a simple comodule, as some of them may give zero-modules. To have more precise information on the simple comodules of $E_{R}$, we need the notion of birank of $R$. Consider the following formal series:

$$
P_{\wedge}(t):=\sum_{i=0}^{\infty} \operatorname{dim} \wedge_{i} t^{i}
$$

We have the following theorem [7] Thm. 3.5]:

1.2. $P_{\wedge}(t)$ is a rational function having negative roots and positive poles:

$$
P_{\wedge}(t)=\frac{\prod_{i=1}^{r}\left(1+x_{i} t\right)}{\prod_{j=1}^{s}\left(1-y_{j} t\right)}, \quad x_{i}, y_{j}>0 .
$$

The pair $(r, s)$ is called the birank of the Hecke symmetry $R$. A partition $\lambda$ determines a non-zero simple $E_{R}$-comodule if and only if $\lambda_{r+1} \leq s$.

The Hecke symmetry $R$ is called even of rank $r$ if it has birank $(r, 0)$, i.e., if the series $P_{\wedge}$ is a polynomial of degree $r$. The Hecke symmetry $R$ is called odd of rank $s$ if it has birank $(0, s)$, i.e., if $P_{\wedge}^{-1}$ is a polynomial of degree $s$. There is generally no relationship between the dimension of $V$ and the (bi)rank of $R$ (see examples below).

Examples. The following are examples of Hecke symmetries that are known so far.

- The solutions of the Yang-Baxter equation of series $A$, due to Drinfel'd and Jimbo [11, provide an example of even Hecke symmetries. The associated quantum groups are called standard deformations of GL( $n)$.

- Cremmer and Gervais [4] found another series of solutions that are also even Hecke symmetries.

- Hecke sums of odd and even Hecke symmetries [5, 16] are examples of noneven, non-odd Hecke symmetries [14].

- Takeuchi and Tambara found a Hecke symmetry that is neither even nor a Hecke sum of an odd and an even Hecke symmetry [18.

- Even Hecke symmetries of rank 2 were classified by Gurevich [5]. He also shows that on each vector space of dimension $\geq 2$, there exists an even Hecke symmetry of rank 2 .

- Hecke symmetries of birank $(1,1)$ were classified by the author $[9]$.

The quantum group of type $A$ is defined to be the "spectrum" of the subsequently defined Hopf algebra. Let $T=\left(t_{i}^{j}\right)$ be a $d \times d$ matrix of new variables. The Hopf 
algebra associated to $R$ is a factor algebra of the free non-commutative algebra over entries of $Z$ and $T$ :

$$
H_{R}:=T\left\langle\left\{z_{j}^{i}, t_{j}^{i}\right\}_{1 \leq i, j \leq d}\right\rangle /\left(z_{m}^{i} z_{n}^{j} R_{k l}^{m n}=R_{p q}^{i j} z_{k}^{p} z_{l}^{q}, t_{p}^{i} z_{j}^{q}=z_{p}^{i} t_{j}^{p}=\delta_{j}^{i}\right) .
$$

$H_{R}$ is a Hopf algebra, the antipode is given by $S\left(z_{j}^{i}\right)=t_{j}^{i}$, and the coquasitriangular structure on $E_{R}$ can be extended to $H_{R}$ thanks to the closedness of $R: r\left(z_{j}^{i}, t_{l}^{k}\right)=$ $P_{j l}^{k i}, r\left(t_{j}^{i}, z_{l}^{i}\right)=R_{l j}^{-1 i k}[8$, Thm. 2.1.1].

If the Hecke symmetry $R$ is even of rank $r, H_{R}$ is cosemisimple and its simple comodules can be parameterized by sequences of $r$ non-decreasing integers 8 , Thm. 3.2.1]. A similar statement holds for odd Hecke symmetries.

If the Hecke symmetry $R$ is neither even nor odd, the structure of $H_{R}$-comodules is more complicated than the structure of $E_{R^{-}}$comodules. In particular, the category $H_{R^{-}}$comod is not semisimple. We have, however, the following result [8] Thm. 2.3.5].

1.3. The natural map $E_{R} \longrightarrow H_{R}$ is injective. Consequently, every simple $E_{R^{-}}$ comodule is a simple $H_{R}$-comodule.

Among $H_{R^{-}}$comodules that are not $E$-comodules, the super determinant plays an important role. The well-known tool for defining the quantum super determinant serves the Koszul complex (of second type) introduced by Manin 17. This is a (bi-)complex, whose $(k, l)$ term is $\wedge_{k} \otimes \mathbf{S}_{l}{ }^{*}$. The differential is induced from the dual basis map. The homology group of this complex is an $H_{R^{-} \text {-comodule; }}$ if it is one dimensional over $k$, it defines a group-like element in $H_{R}$ called the homological determinant or quantum super determinant or, in some cases, the quantum Berezinian.

\section{The Koszul Complex}

We begin with the description of the Koszul complex. We first recall the dual comodule of a tensor product of two comodules. For two (finite-dimensional) comodules $V, W$, the dual to $V \otimes W$ is isomorphic to $W^{*} \otimes V^{*}$, with the pairing given by $(\varphi \otimes \psi)(v \otimes w):=\varphi(w) \psi(v), \varphi \in W^{*}, \psi \in V^{*}, v \in V, w \in W$. The dual to longer tensor products is defined in a similar way.

Fix a basis $x_{1}, x_{2}, \ldots, x_{d}$ of $V$ and let $\xi^{1}, \xi^{2}, \ldots, \xi^{d}$ be the dual basis in $V^{*}$. Let $\mathrm{ev}_{V}$, be the evaluation map $\operatorname{ev}_{V}(\varphi \otimes v)=\varphi(v)$ and $\mathrm{db}_{V}$ be the dual basis map defined as follows: $\mathrm{db}: k \longrightarrow V \otimes V^{*}, \mathrm{db}(1)=\sum_{i} x_{i} \otimes \xi^{i}$. These maps clearly do not depend on the choice of basis and are maps of $H_{R^{-c o m o d u l e s . ~ T h e ~ t e r m ~} K^{k, l}}$ of the Koszul complex associated to $R$ is $\wedge_{k} \otimes S_{l}{ }^{*}$, and the differential $d_{k, l}$ is given by:

$$
\wedge_{k} \otimes \mathrm{S}_{l}^{*} \rightarrow V^{\otimes l} \otimes V^{* \otimes l} \stackrel{\mathrm{id} \otimes \mathrm{db}_{V}}{\longrightarrow}{ }^{\otimes \mathrm{id}} V^{\otimes k+1} \otimes V^{* \otimes l+1} \stackrel{Y_{k+1} \otimes X_{l+1}^{*}}{\longrightarrow} \wedge_{k+1} \otimes \mathrm{S}_{l+1}{ }^{*},
$$

where $X_{l}, Y_{k}$ are the $q$-symmetrizer operators introduced in the previous section. Thus we have in fact a collection of diagonal subcomplexes, each of which contains the terms $K^{k, l}$ with $k-l$ equal to a fixed number. One defines another differential $d^{\prime}$ as follows:

$\bigwedge_{k} \otimes \mathrm{S}_{l}{ }^{*} \rightarrow V^{\otimes l} \otimes V^{* \otimes l} \stackrel{\mathrm{id} \otimes \mathrm{ev}_{V} \tau_{V \otimes} V^{*} \otimes \mathrm{id}}{\longrightarrow} V^{\otimes k-1} \otimes V^{* \otimes l-1} \stackrel{Y_{k-1} \otimes X_{l-1}^{*}}{\longrightarrow} \wedge_{k-1} \otimes \mathrm{S}_{l-1}{ }^{*}$,

where $\tau_{V, V^{*}}$ denotes the braiding on $V \otimes V^{*}$ induced from the coquasitriangular structure on $H_{R}$, its matrix is given by $P$, the inverse to the half-adjoint of $R$. 


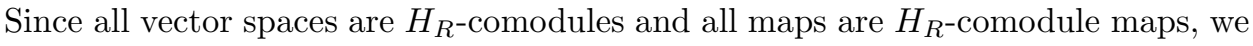
have in fact complexes in $H_{R}$-comod.

The differentials $d$ and $d^{\prime}$ satisfy [5]

$$
\left.\left(q d d^{\prime}+d^{\prime} d\right)\right|_{K^{k, l}}=q^{k}\left(\operatorname{rank}_{q} R+[l-k]_{q}\right) \text { id, }
$$

where $\operatorname{rank}_{q} R:=P_{i j}^{i j}$, and $P$ is given in (1). Hence, if $\operatorname{rank}_{q} R \neq-[l-k]_{q}$, the cohomology group at the term $(k, l)$ vanishes.

Theorem 1. Let $R$ be a Hecke symmetry of birank $(r, s)$. Then

(i) $\operatorname{rank}_{q} R=-[s-r]_{q}$;

(ii) the simple comodule $I_{\lambda}$ is injective and projective in the category of $H_{R^{-}}$ comodules if and only if $\lambda_{r} \geq s$;

(iii) the homology of the Koszul complex at the term $(r, s)$ is non-vanishing.

Proof. Since $R$ has birank $(r, s)$, the simple $E$-comodule $I_{\lambda} \neq 0$ iff $\lambda_{r+1} \leq s$. Using this fact and the Littlewood-Richardson formula, we can easily show that (Hom means $\operatorname{Hom}^{H_{R}}$ ):

$\operatorname{Hom}\left(I_{\left((s+1)^{r}\right)}, I_{\left(s^{r+1}\right)} \otimes \wedge_{r} \otimes \mathrm{S}_{s}{ }^{*}\right) \cong \operatorname{Hom}\left(I_{\left((s+1)^{r}\right)} \otimes \mathrm{S}_{s}, I_{\left(s^{r+1}\right)} \otimes \wedge_{r}\right)=k$, $\operatorname{Hom}\left(I_{\left((s+1)^{r}\right)}, I_{\left(s^{r+1}\right)} \otimes \wedge_{r-1} \otimes \mathrm{S}_{s-1}{ }^{*}\right) \cong \operatorname{Hom}\left(I_{\left((s+1)^{r}\right)} \otimes \mathrm{S}_{s-1}, I_{\left(s^{r+1}\right)} \otimes \wedge_{r-1}\right)=0$, $\operatorname{Hom}\left(I_{\left((s+1)^{r}\right)}, I_{\left(s^{r+1}\right)} \otimes \wedge_{r+1} \otimes \mathrm{S}_{s+1}{ }^{*}\right) \cong \operatorname{Hom}\left(I_{\left((s+1)^{r}\right)} \otimes \mathrm{S}_{s+1}, I_{\left(s^{r+1}\right)} \otimes \wedge_{r+1}\right)=0$.

As a consequence, $I_{\left(s^{r+1}\right)} \otimes \wedge_{r} \otimes \mathrm{S}_{s}{ }^{*}$ contains $I_{\left((s+1)^{r}\right)}$ as a subcomodule while the comodules $I_{\left(s^{r+1}\right)} \otimes \wedge_{r-1} \otimes \mathrm{S}_{s-1}{ }^{*}, I_{\left(s^{r+1}\right)} \otimes \wedge_{r+1} \otimes \mathrm{S}_{s+1}{ }^{*}$ do not.

Assume that $\operatorname{rank}_{q} R \neq-[s-r]_{q}$. Then the complex is exact at $K^{r, s}$ and $d d^{\prime}+d^{\prime} d=q^{r}\left(\operatorname{rank}_{q} R+[s-r]_{q}\right)$ id $\neq 0$. On the other hand, since $I_{(s+1)^{r}}$ is a submodule of $I_{\left(s^{r+1}\right)} \otimes \wedge_{r+1} \otimes \mathrm{S}_{s+1}{ }^{*}$, the restriction of $\operatorname{id}_{I_{\left((s+1)^{r}\right)}} \otimes d^{r, s}$ to it should be zero. Analogously, the restriction of $\operatorname{id}_{I_{\left((s+1)^{r}\right)}} \otimes d^{m, n}$ to $I_{\left((s+1)^{r}\right)}$ is zero. Thus, the restriction of $d d^{\prime}+d^{\prime} d$ on $I_{(s+1)^{r}}$ is zero, a contradiction. Therefore, $\operatorname{rank}_{q} R=-[s-r]_{q}$.

According to [10, Thm. 3.2] if $\operatorname{rank}_{q} R=-[s-r]_{q}$, then $H$ possesses a non-zero integral (i.e. an $H$-comodule homomorphism $H \longrightarrow k$, where $H$ coacts on itself by the coproduct and on $k$ by the unit map). Then, according to [10, Prop. 5.1] and to [9, Thm. 3.1], $I_{\lambda}$ is injective and projective in $H$-comod iff $\lambda_{r} \geq s$. Thus, $I_{\left((s+1)^{r}\right)}$ is projective and injective. Therefore, if $I_{\left((s+1)^{r}\right)}$ is a subquotient of a comodule, it is a direct summand; hence it cannot be a subquotient of $I_{\left(s^{r+1}\right)} \otimes \wedge_{m-1} \otimes \mathrm{S}_{n-1}{ }^{*}$, and in particular, it cannot be a subcomodule of $I_{\left(s^{r+1}\right)} \otimes \operatorname{Im} d^{r-1, s-1}$. Consequently,

$$
I_{\left(s^{r+1}\right)} \otimes \operatorname{Im} d^{r-1, s-1} \neq I_{\left(s^{r+1}\right)} \otimes \operatorname{Ker} d^{m, n} .
$$

Thus, the sequence

$\cdots \rightarrow I_{\left(s^{r+1}\right)} \otimes \wedge_{r-1} \otimes \mathrm{S}_{s-1}{ }^{*} \rightarrow I_{\left(s^{r+1)}\right)} \otimes \wedge_{r} \otimes \mathrm{S}_{s}{ }^{*} \rightarrow I_{\left(s^{r+1}\right)} \otimes \wedge_{r+1} \otimes \mathrm{S}_{r+1}{ }^{*} \rightarrow \cdots$, which is obtained by tensoring $K^{*}$ with $I_{\left(s^{r+1}\right)}$, is not exact at the term $(r, s)$, whence neither is $K^{*}$.

\section{THE CASE $q=1$}

Assume in this section that $q=1$; thus, $R^{2}$ is the identity map and $H$-comod is a tensor category (i.e., symmetric rigid monoidal). By a theorem of Deligne [1], there exists a faithful and exact tensor (i.e. symmetric monoidal) functor $\mathcal{F}$ from $H$-comod to the category of vector superspaces. Under this functor, $V$ is mapped 
to a certain vector superspace $\bar{V}$ and $R$ is mapped to the supersymmetry on $\bar{V} \otimes \bar{V}$, denoted by $T$.

We can therefore reconstruct a super bialgebra $\bar{E}$ and a Hopf super algebra $\bar{H}$ from $\bar{V}$ and $T$. We will show that this Hopf superalgebra is isomorphic to the function algebra over the general linear supergroups $G L(r \mid s)$, where $(r, s)$ is the birank of $R$, or, in other words, the super dimension of $\bar{V}$ is $(r \mid s)$. Indeed, $\bar{E}$ is the function algebra on $\operatorname{End}(\bar{V})$ and the images of $I_{\lambda}$ under the embedding $\mathcal{F}$ are simple $\bar{E}$-comodules. Since $\mathcal{F}$ is faithful and exact and since $I_{\lambda} \neq 0 \Leftrightarrow \lambda_{r+1}<s$, we conclude that $\bar{E}$ is isomorphic to the function algebra on $M(r \mid s)$. Hence $\bar{H}$ is isomorphic to the function algebra on $G L(r \mid s)$, by virtue of 1.3 .

Let $\bar{K}$ " denote the image of the complex $K^{*}$. Then the homology of $\bar{K}{ }^{*}$ is concentrated at the term $(r, s)$, and is one-dimensional; it defines the super determinant. As a consequence, the homology of $K^{*}$ is also concentrated at the term $(r, s)$, for $\mathcal{F}$ is faithful and exact. Let $D$ denote the homology of $K^{*}$. Then $\bar{D}$, the image of $D$ under $\mathcal{F}$, is one-dimensional and hence invertible; consequently,

$$
\mathcal{F}\left(D^{*} \otimes D\right) \cong \mathcal{F}\left(D^{*}\right) \otimes \mathcal{F}(D) \cong \bar{D}^{*} \otimes \bar{D} \cong k,
$$

where the last isomorphism is given by the evaluation morphism, that is, the image of $\operatorname{ev}_{D}: D^{*} \otimes D \longrightarrow k$ under $\mathcal{F}$. Since $\mathcal{F}$ is faithful and exact, we conclude that $D^{*} \otimes D \cong k$, that is $D$ is invertible, hence one-dimensional. Thus, we have proved:

Theorem 2. Let $R$ be a vector symmetry of birank $(r, s)$. Then the associated Koszul complex is exact everywhere except at the term $(r, s)$ where it has a onedimensional homology group, which determines a group-like element called the homological determinant.

\section{THe CASE $q$ GENERIC}

Using the result of the previous section we show in this section that given a Hecke symmetry of birank $(r, s)$ that depends algebraically on $q$, then, for a dense set of values $q$, the associated Koszul complex is exact everywhere except at the term $(r, s)$, where it has a one-dimensional homology group and thus determines a group-like element in $H_{R}$, called the homological determinant. In this section $k$ will be assumed to be the field $\mathbb{C}$ of complex numbers.

Thus let $R=R_{q}$ be a Hecke symmetry depending on a parameter $q \in \mathbb{C}$. We first observe that the dimension of $\wedge_{q, k}$ does not depend on $q$, so far as $q$ is not a root of unity. Indeed, $\wedge_{q, k}$ is the image of a projection, and its dimension can be given as the trace of a matrix that depends algebraically on $q$. Since $\mathbb{C}$ without the set of roots of unity is still connected, we conclude that this trace, being always integral, must be a constant. The same happens with $\mathrm{S}_{q, l}$. Thus, the terms of $K^{*}$ have dimension not depending on $q$.

On the other hand, observe that the rank of the operator $d_{q}^{k, l}$, for almost any $q$ (that is, except for a finite number of values of $q$ ) is larger than the rank of $d_{1}^{k, l}$ and for the kernel of $d_{q}^{k, l}$ we have the reversed inequality. Consequently, the dimension over $k$ of the homology group $H\left(K_{q}^{k, l}\right)$ for almost any $q$ is less than or equal to the dimension of $H\left(K_{q}^{k, l}\right)$. According to Theorems 1 and 2, we conclude that for an algebraically dense set of values of $q, H\left(K_{q}^{k, l}\right)=0$, for all $(k, l) \neq(r, s)$ and $H\left(K_{q}^{r, s}\right)$ is one-dimensional. 
Theorem 3. Let $R=R_{q}$ be a Hecke symmetry over $\mathbb{C}$, depending algebraically on $q$. Then there is an algebraically dense set of values of $q$ for which the homology of the Koszul complex is one-dimensional and concentrated at the term $(r, s)$, where $(r, s)$ is the birank of $R$.

\section{REFERENCES}

[1] P. Deligne. Catégories tensorielles. Mosc. Math. J., 2(2):227-248, 2002 . MR 1944506 (2003k:18010)

[2] R. Dipper and G. James. Representations of Hecke Algebras of General Linear Groups. Proc. London Math. Soc., 52(3):20-52, 1986. MR0812444 (88b:20065)

[3] R. Dipper and G. James. Block and Idempotents of Hecke Algebras of General Linear Groups. Proc. London Math. Soc., 54(3):57-82, 1987. MR0872250 (88m:20084)

[4] E.Cremmer and J.-L.Gervais. The Quantum Groups Structure Associated With Nonlinearly Extended Virasoro Algebras. Comm. Math. Phys., 134:619-632, 1990. MR1086746 (92a:81072)

[5] D.I. Gurevich. Algebraic Aspects of the Quantum Yang-Baxter Equation. Leningrad Math. Journal, 2(4):801-828, 1991. MR1080202 (93e:17018)

[6] P.H. Hai. Koszul Property and Poincaré Series of Matrix Bialgebra of Type $A_{n}$. J. of Algebra, 192(2):734-748, 1997. MR1452685 (98g:16006)

[7] P.H. Hai. Poincaré Series of Quantum Spaces Associated to Hecke Operators. Acta Math. Vietnam, 24(2):236-246, 1999. MR1710780 (2000j:16048)

[8] P.H. Hai. On Matrix Quantum Groups of Type $A_{n}$. Int. J. of Math., 11(9):1115-1146, 2000. MR $1809304(2001 \mathrm{~m}: 16064)$

[9] P.H. Hai. Splitting comodules over Hopf algebras and application to representation theory of quantum groups of type $A_{0 \mid 0}$. J. of Algebra, 245(1):20-41, 2001. MR1868181 (2002j:16045)

[10] P.H. Hai. The integral on quantum super groups of type $A_{r \mid s}$. Asian J. of Math., 5(4):751770, 2001. MR1913820 (2003g:20082)

[11] M. Jimbo. A q-analogue of $\mathrm{U}(\mathfrak{g} l(N+1))$, Hecke algebra, and the Yang-Baxter equation. Lett. Math. Phys., 11:247-252, 1986. MR0841713 (87k:17011)

[12] R. Larson and J. Towber. Two Dual Classes of Bialgebras Related To The Concepts of "Quantum Groups" and "Quantum Lie Algebra". Comm. in Algebra, 19(12):3295-3345, 1991. MR 1135629 (93b:16070)

[13] V.V. Lyubashenko. Hopf Algebras and Vector Symmetries. Russian Math. Survey, 41(5):153154, 1986. MR0878344(88c:58007)

[14] V.V. Lyubashenko and A. Sudbery. Quantum Super Groups of GL $(n \mid m)$ Type: Differential Forms, Koszul Complexes and Berezinians. Duke Math. Journal, 90:1-62, 1997. MR1478542 (98i:16041)

[15] I.G. Macdonald. Symmetric functions and the Hall polynomials. Oxford University Press, New York, 1979 (Second edition 1995). MF1354144 (96h:05207)

[16] S. Majid and M. Markl. Glueing Operation for R-Matrices, Quantum Groups and LinkInvariants of Hecke Type. Math. Proc. Camb. Philos. Soc., 119(1):139-166, 1996. MR1356165 (96i:17015)

[17] Yu.I. Manin. Gauge Field Theory and Complex Geometry. Springer-Verlag, 1988. MR 0954833 (89d:32001)

[18] M. Takeuchi and D. Tambara. A new one-parameter family of $2 \times 2$ quantum matrices. Hokkaido Math. Journal, XXI(3):409-419, 1992. See also Proc. Japan. Acad., 67, no. 8, 267-269. 1991. MR1191027|(93j:17036): MR1137925 (93b:17054)

Institute of Mathematics, P.O. Box 631, 10000 Boho, Hanoi, Vietnam

E-mail address: phung@math.ac.vn

Current address: FB6 Mathematik, Universität Duisburg-Essen, 45117 Essen, Germany

E-mail address: ho-hai.phung@uni-essen.de 